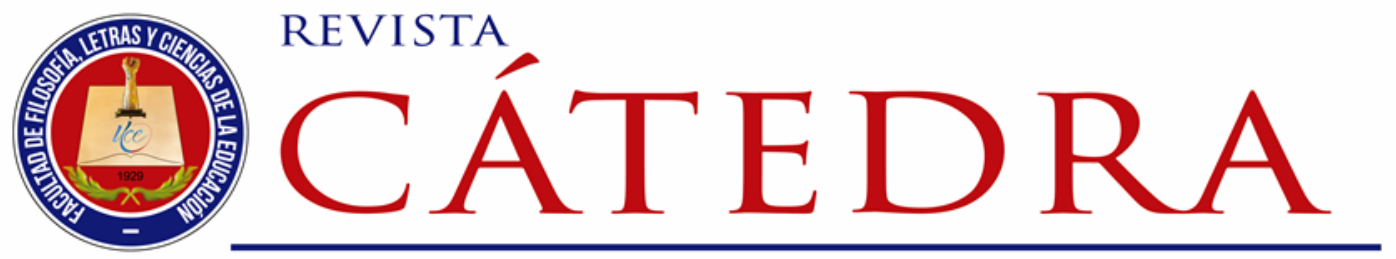

\title{
Perspectivas de la producción científica en las universidades del Ecuador
}

\section{Perspectives of scientific production in the universities of Ecuador}

\author{
Jorge Balladares-Burgos \\ Universidad Andina Simón Bolívar, Quito, Ecuador \\ jorge.balladares@uasb.edu.ec \\ https://orcid.org/0000-0001-7033-1970 \\ Ángela García-Naranjo \\ Universidad Central del Ecuador, Quito, Ecuador \\ aegarcian@uce.edu.ec \\ https://orcid.org/0000-0001-9299-8282 \\ Cristina Granda-Villamar \\ Universidad Central del Ecuador, Quito, Ecuador \\ bcgranda@uce.edu.ec \\ https://orcid.org/0000-0003-3799-1365
}

(Recibido: 09/03/2020; Aceptado: 11/03/2020; Versión final recibida: 25/04/2020)

Cita del artículo: Balladares-Burgos, J. García-Naranjo, A. Granda-Villamar, C. (2020). Perspectivas de la producción científica de las universidades del Ecuador. Revista Cátedra, 3(2), 126-149.

\section{Resumen}

El presente artículo surge de la necesidad de conocer la situación de la producción científica en índices nacionales e internacionales de las universidades ecuatorianas desde el año 2003 al 2017. Estos índices revelan la desventaja de las universidades a nivel nacional e internacional. Las Instituciones de Educación Superior (IES) ecuatorianas se encuentran en puestos inferiores a nivel Iberoamericano (IBE), Latinoamericano y el Caribe (LAC) con respecto al número de publicaciones que han realizado. La investigación sobre los índices de creación científica de las IES del Ecuador permite vislumbrar el estado actual de la problemática. Además, en 2008 el Gobierno de Ecuador implementa una reforma de

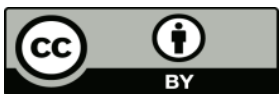


Educación Superior para incentivar la creación científica. Por consiguiente, el propósito del estudio es analizar el estado de producción científica entre las diez mejores universidades ecuatorianas, a nivel nacional e internacional desde el año 2003 al 2017. Asimismo, determinar la influencia de la reforma de Educación Superior del 2008 en cuanto a la producción científica de las IES. La metodología empleada en esta investigación es de carácter inductivo-deductivo, apoyada en la revisión bibliográfica y la hermenéutica. Se concluye que en el período 2003-2013 la Universidad San Francisco de Quito publicó más contenido científico a nivel nacional mientras que en 2013-2017 fue la Universidad de las Fuerzas Armadas. También, se evidencia que las universidades subieron su producción investigativa a nivel IBE, LAC y nacional. Con estos datos se busca evidenciar los efectos de la implementación de políticas públicas que regulen la producción científica en las IES.

\section{Palabras Clave}

Escritura científica, índices, normativa, universidad.

\section{Abstract}

This article arises from the need to know the situation of scientific production in national and international indices of Ecuadorian universities from 2003 to 2017. These indices show the disadvantage of universities at the national and international level. Ecuadorian Higher Education Institutions (HEIs) are in lower positions at the Ibero-American (IBE), Latin American and Caribbean (LAC) level with respect to the number of publications they have made. Research on the scientific creation indices of HEIs in Ecuador allows to glimpse the current state of the problem. In addition, in 2008 the Government of Ecuador implements a reform of Higher Education to encourage scientific creation. Therefore, the purpose of the study is to analyze the state of scientific production among the top Ten Ecuadorian Universities, nationally and internationally from 2003 to 2017. Likewise, to determine the influence of the 2008 Higher Education reform regarding the scientific production of HEIs. The methodology used in this research is inductive-deductive, supported by literature review and hermeneutics. It is concluded that in the period 2003-2013 the San Francisco de Quito University published more scientific content at the national level, while in 2013-2017 it was the University of the Armed Forces. Also, it is evident that the universities raised their research production at IBE, LAC and national levels. With these data we seek to demonstrate the effects of the implementation of public policies that regulate scientific production in HEIs.

\section{Keywords}

Writing scientist, index, regulations, university.

\section{Introducción}

El nivel de producción científica se refiere a la capacidad que tienen las personas, instituciones y organismos que forman parte de la sociedad para divulgar información sobre alguna investigación de un tema específico. Esta labor investigativa ha sido determinada como una de las actividades académicas que las Instituciones de Educación Superior (IES) deben promover en sus docentes y futuros profesionales. En los últimos años se han realizado investigaciones sobre el número de publicaciones científicas que las IES del Ecuador han realizado. Estas han revelado una escasa elaboración de artículos científicos entre las IES ecuatorianas a nivel nacional e internacional. Así mismo, han denotado una escasa aportación al campo científico y al desarrollo de la sociedad. Castillo y Powel (2019) mencionan que esta problemática pudo haber sido generado por "la falta de cultura 
científica y políticas adecuadas que promuevan la investigación, además del descuido en priorizar la investigación en las universidades" (p.1). Es decir, la carente producción científica del país se dio gracias a que no existían organismos que regulen la investigación científica dentro de las IES antes del año 2008. También, se le atribuye a que el Estado no destinaba fondos monetarios al área de investigación. Finalmente, dentro de las IES, los estudiantes e investigadores no poseían una cultura de investigación.

El Estado ecuatoriano para tratar de incrementar los índices de producción científica en sus universidades implementó en el año 2008 la reforma de Educación Superior. Al mismo tiempo, creó un conjunto de estrategias y proyectos para mejorar la situación investigativa en el país. No obstante, el conocimiento del impacto de las reformas y estrategias implementadas con respecto a la producción científica aún no ha sido profundamente estudiadas. Existen muy pocos artículos donde se analicen el número de publicaciones científicas de las principales IES del Ecuador desde el año 2003 hasta la actualidad. Por consiguiente, es necesario realizar una búsqueda exhaustiva de fuentes bibliográficas que brinden información actualizada y pertinente a la investigación que se pretende realizar.

Los informes publicados por SCImago Institutions Rankings (SIR) presentan la producción y aporte científico de las IES a nivel Latinoamericano, Iberoamericano y del Caribe. En consecuencia, se ha podido obtener datos concretos en cuanto a la producción científica con respecto a las IES del Ecuador. Este rainking "ofrece una clasificación de las instituciones según el número de trabajos indexados en Scopus (...) con base en tres factores fundamentales: investigación, innovación e impacto social" (SIR Iber, 2019, p.4). Para la elaboración de esta investigación se ha considerado el componente de investigación que permite conocer el número de publicaciones científicas ha realizado cada universidad según los períodos mencionados en el informe.

Este componente mide "la capacidad institucional de generar productos científicos y difundirlos a través de canales reconocidos de comunicación científica" (SIR Iber, 2019, p. 24). Sin embargo, una dificultad presentada en el proceso de investigación fue que los datos encontrados en el SIR Iber corresponden a la producción científica de "Andorra, España, Portugal, y los países de Latinoamérica” (SIR Iber, 2019, p.5). Razón por la cual fue necesario extraer sólo la información referente a las IES del Ecuador tomando en cuenta las diez mejores universidades del país.

El problema de la escasa divulgación científica que las IES ecuatorianas ha incidido en el puesto que ocupan a nivel Iberoamericano (IBE), Latinoamericano y el Caribe y a nivel nacional. Según Witter (citado por Piedra y Martínez, 2007) la producción científica es:

La forma mediante la cual una universidad o institución de investigación
se hace presente a la hora de hacer ciencia, es una base para el
desenvolvimiento y la superación de dependencia entre países y regiones
de un mismo país; es un vehículo para la mejoría de la calidad de vida de
los habitantes de un país, es una forma de hacerse presente no solo hoy,
sino también mañana (p.34).

Por tanto, la situación en la que se encontraban las universidades ecuatorianas antes del año 2008 ha impedido que puedan alcanzar puestos relevantes dentro de la producción científica a nivel nacional e internacional. Por otra parte, el bajo nivel de investigaciones evitó que las Instituciones de Educación Superior pueda formar parte de las soluciones a ciertas problemáticas en la sociedad ecuatoriana o mundial.

Licencia Creative Commons Atribución 4.0 Internacional (CC BY 4.0)

Revista Cátedra, 3(2), pp. 126-149, mayo-agosto 2020. e-ISSN: 2631-2875

https://doi.org/10.29166/10.29166/catedra.v3i2.2183 
En consecuencia, la relevancia de este artículo reside en la investigación que se pretende realizar sobre los índices de creación científica dentro de las diez mejores universidades ecuatorianas desde los años 2003 al 2017. Esto permitirá conocer el papel que tienen las universidades ecuatorianas a nivel Iberoamericano (IBE), Latinoamericano y el Caribe y a nivel nacional en el desarrollo e incremento de conocimientos que permitan la mejora de la sociedad. El estudio sobre esta problemática ayuda a revelar nuevas perspectivas de los cambios que han ocurrido con respecto a la producción científica y devela el impacto de la intervención del Estado.

La intención de esta investigación es demostrar que la producción científica de las diez principales universidades ecuatorianas se ha elevado considerablemente durante los últimos años. Todo esto gracias a la reforma y a las políticas públicas establecido por el Gobierno nacional y los distintos organismos de regulación y control. Las diez mejores universidades, según QS World University Rankings 2020, comparadas fueron: Universidad San Francisco de Quito, Escuela Superior Politécnica del Litoral, Pontificia Universidad Católica del Ecuador, Escuela Politécnica Nacional, Universidad Central del Ecuador, Universidad de Cuenca, Universidad de las Fuerzas Armadas, Universidad Técnica Particular de Loja, Universidad de Especialidades Espíritu Santo, Universidad de las Américas.

El propósito de este artículo es analizar el estado de producción científica entre las diez mejores universidades ecuatorianas, a nivel nacional e internacional durante los períodos 2003-2008, 2006-2010, 2009-2013 y 2013-2017 con base en los resultados del SIR Iber. Con este fin, se planea encontrar respuestas a:

- ¿Cuál fue el número de publicaciones científicas durante los períodos 2003-2008, 2006-2010, 2009-2013 y 2013-2017 de cada una de las diez IES?

- ¿Qué IES lideraron el índice de producción científica durante esos períodos?

- ¿Existió un incremento en el número de publicaciones científicas a partir de la Reforma de Educación Superior?

Asimismo, se busca determinar la influencia de la reforma de Educación Superior del 2008 en la creación científica.

En cuanto a la estructura y contenido del artículo, en la sección 2 se presenta los conceptos relacionados con la investigación. En la sección 3, la revisión de las diversas fuentes bibliográficas de experiencias y resultados de investigaciones relacionadas a la producción científica de las universidades del Ecuador. En la sección 4, se especifica la metodología utilizada para desarrollar esta investigación. En la sección 5, se muestran los resultados de la producción científica de las 10 mejores universidades del Ecuador durante los períodos 2003-2008, 2006-2010, 2009-2013 y 2013-2017 con base en los resultados del SIR Iber y la producción científica antes y después de la reforma de educación superior de 2008. En la sección 6, se establecen las conclusiones de acuerdo con los resultados obtenidos.

\section{Conceptos relacionados}

\subsection{Horizontes epistemológicos sobre la producción científica dentro del Ecuador}

Desde los inicios de la universidad en el Ecuador hasta el año 2000 aproximadamente, el Sistema de Educación Superior ha sufrido varios cambios. El más significativo con respecto a la calidad educativa surge en el 2002 con la aparición del Consejo de Evaluación y Acreditación de la Educación Superior del Ecuador (CONEA). Institución que se preocupó en evaluar la situación de la investigación y producción científica dentro de las IES en el

Licencia Creative Commons Atribución 4.0 Internacional (CC BY 4.0)

Revista Cátedra, 3(2), pp. 126-149, mayo-agosto 2020. e-ISSN: 2631-2875

https://doi.org/10.29166/10.29166/catedra.v3i2.2183 
Ecuador. Los horizontes epistemológicos relacionados a la producción científica se pueden comprender iniciando con un breve repaso de la historia del Sistema de Educación Superior en el Ecuador.

La existencia de un Sistema de Educación Superior tiene sus orígenes en los años de 1596 con la creación de la Universidad de San Fulgencio a cargo del Clero, cuya "principal finalidad era la educación de los criollos" (Pacheco y Pacheco, 2015, párr. 7). En esta etapa del sistema de educación superior la prioridad era educar a las personas de familias prestigiosas y españoles radicados en América. La educación superior en ese entonces fue el privilegio de pocos. Principalmente estaba orientada a las familias más aristócratas del país y en aquellos hombres que veían en el sacerdocio una forma de vida.

En los años de 1822 a 1830 con el inicio de la República Independiente la Universidad de San Fulgencio pasaría a ser la Universidad Central del Ecuador y para 1869 se crea la Escuela Politécnica Nacional (EPN) cuyo fin fue "la formación acorde a las necesidades prácticas y técnicas del país"(Pacheco y Pacheco, 2015, párr. 8). Esta institución fue fundada en la presidencia de García Moreno y fue muy bien acogida como el primer centro de docencia e investigación científica del país. Con la EPN el Ecuador vería la posibilidad de desarrollo técnico e industrial.

Después de varios años, ya a comienzos del siglo XX con la llegada de la Revolución Industrial, el Ecuador cambia totalmente de visión y procura la "incorporación de los ciudadanos a la producción y desarrollo social a través del estudio de las ciencias, la práctica y la investigación" (Pacheco y Pacheco, 2015, párr.9). En las décadas de 1930 y 1940, en el Ecuador se expide la Ley de Educación Superior la cual permite a las universidades independizarse técnica y administrativamente del Estado. En 1998 se redacta la Constitución Política del Ecuador en la cual gracias a los políticos de corte neoliberal se privatiza el acceso a la educación de tercer nivel.

Después de tantos años de historia universitaria, en el año 2002 entra en funciones el Consejo Nacional de Evaluación y Acreditación de las Universidades del Ecuador (CONEA). Esta entidad pública y de acuerdo con la Ley Orgánica de Educación Superior expedida en el 2000 realiza un primer acercamiento a la evaluación de la calidad educativa en las Instituciones de Educación Superior (IES).

\subsection{Producción científica del Ecuador respecto a la normativa de organismos de control de las Instituciones de Educación Superior}

La normativa busca el aumento de producción científica por lo que una de sus políticas es determinar que los recursos tanto de universidades privadas y públicas sean destinados para desarrollar investigaciones y con ello la divulgación de resultados. Por ejemplo, en el artículo 35 se establece que: "las instituciones del Sistema de Educación Superior podrán acceder adicional y preferentemente a los recursos públicos concursables de la pre asignación para investigación, ciencia, tecnología e innovación establecida en la Ley correspondiente" (LOES, 2010a, p. 21). Es decir, el estado se compromete con las universidades a brindar recursos económicos que cubran los gastos de proyectos investigativos. Para la asignación de los recursos económicos el estado tomará en cuenta el criterio de evaluación excelencia académica que evalúa el CACES. De acuerdo con este criterio las IES recibirán entre el 3 y $10 \%$ del monto total.

Las universidades en esta ley también se comprometen a destinar un porcentaje de los recursos dados para publicaciones de investigaciones. Esto se estipula en el artículo 36 de la siguiente forma:

Licencia Creative Commons Atribución 4.0 Internacional (CC BY 4.0)

Revista Cátedra, 3(2), pp. 126-149, mayo-agosto 2020. e-ISSN: 2631-2875

https://doi.org/10.29166/10.29166/catedra.v3i2.2183 
Asignación de recursos para publicaciones, becas para profesores o profesoras e investigaciones.- Las instituciones de educación superior de carácter público y particular asignarán obligatoriamente en sus presupuestos, por lo menos, el seis por ciento (6\%) a publicaciones indexadas, becas de posgrado para sus profesores o profesoras e investigaciones en el marco del régimen de desarrollo nacional. La Secretaría Nacional de Educación Superior, Ciencia, Tecnología e Innovación velará por la aplicación de esta disposición (LOES, 2010a, p.21).

En este artículo se evidencia los incentivos económicos que se dan a los investigadores y docentes para que puedan desarrollar su labor. Además, la responsabilidad que tiene la Secretaría Nacional de Educación Superior, Ciencia, Tecnología e Innovación para que esto pueda cumplirse. No obstante, estos no son los únicos apartados que refieren al impulso de producción científicas existen durante toda la Ley Orgánica de Educación Superior un sin número de artículos que intentan promover la actividad investigativa. Por ejemplo, fomentar programas de carácter científico con el fin de promover el desarrollo del país.

Las responsabilidades dadas a las Instituciones de Educación Superior con respecto a la investigación científica además del constante control que el Estado debe tener sobre estas. Es así que el Sistema de Educación superior debe establecer organismos para que controlen la calidad de las universidades. En el artículo 353 se expresa que:

1. Un organismo público de planificación, regulación y coordinación interna del sistema y de la relación entre sus distintos actores con la Función Ejecutiva.

2. Un organismo público técnico de acreditación y aseguramiento de la calidad de instituciones, carreras y programas, que no podrá conformarse por representantes de las instituciones objeto de regulación (Constitución de la República del Ecuador, 2008 a,159).

Entre uno de los puntos que tomaran los organismos para medir la calidad educativa del tercer nivel de educación será la generación de investigaciones de cada universidad esto permite que el artículo en un principio mencionado sea ejecutado. Es así como nacen organismos como el CACES, la SENESCYT y el CES que regularan las Instituciones de Educación Superior ecuatorianas. Estas, también, implementaran normas que obliguen a las universidades a tomar a la producción científica como trabajo prioritario de la vida académica.

\subsubsection{Consejo de Aseguramiento de la Calidad de la Educación Superior (CACES)}

Para garantizar la calidad de la educación superior el Estado ha promovido la creación de organismos que se encarguen de evaluar, acreditar y promover la calidad del Sistema de Educación Superior (SES) en el Ecuador. Para alcanzar este fin se encuentra el Consejo de Aseguramiento de la Calidad de la Educación Superior (CACES).

Este organismo instaurado por el Estado inicia sus funciones en 1989 con el nombre del Consejo Nacional de Universidades y Escuelas Politécnicas (CONUEP). El CONUEP llevó a cabo el proceso de evaluación, el mismo que concluyó que "en Ecuador no existe un sistema de educación superior [...] en sentido estricto. Lo que hay es un conjunto de instituciones universitarias independientes unas de otras y que a nivel administrativo tienen ciertos mecanismos de cooperación [...]" (CONUEP 1992, p. 29, como cita CACES, 2018, p.11).

Licencia Creative Commons Atribución 4.0 Internacional (CC BY 4.0)

Revista Cátedra, 3(2), pp. 126-149, mayo-agosto 2020. e-ISSN: 2631-2875

https://doi.org/10.29166/10.29166/catedra.v3i2.2183 
Después de este proceso de evaluación realizado por el CONESUP, en el año 2002 este organismo toma el nombre de Consejo Nacional de Evaluación y Acreditación (CONEA), el mismo que emprende un nuevo proceso de evaluación y acreditación de IES de todo el país. Este organismo para continuar con sus labores, en el año 2011, toma el nombre de Consejo de Evaluación, Acreditación y Aseguramiento de la Calidad de la Educación Superior (CEAACES). Este organismo sigue con la tarea de llevar a cabo los procesos de evaluación, acreditación y aseguramiento de la calidad en IES del país.

Finalmente, en el año 2018 este organismo recibe el nombre de Consejo de Aseguramiento de la Calidad de la Educación Superior (CACES). Este organismo según la página web oficial del CACES (2019) se encarga de:

Regular, coordinar y planificar los procesos participativos de acompañamiento, evaluación, acreditación y cualificación para garantizar el desarrollo de una cultura de la calidad en las instituciones de educación superior, enfocada en el equilibrio de la docencia, la investigación e innovación y la vinculación con la sociedad (p.1).

En cualquiera que sea el caso todos los organismos anteriormente nombrados han tenido la labor de realizar procesos de evaluación orientados a garantizar la calidad educativa en todos las Instituciones de Educación Superior del Ecuador.

\subsubsection{Normativa del Consejo de Aseguramiento de la Calidad de la Educación Superior (CACES) con respecto a la producción científica de la Instituciones de Educación Superior}

El CONEA en el 2002 (actual CACES) inicia el proceso de evaluación de las IES del país basándose en cinco criterios: academia, eficiencia académica, organización, infraestructura e investigación. Dentro del criterio de investigación se evaluó "el nivel alcanzado en la generación de conocimientos científicos" (Pacheco y Pacheco, 2015, secc. La nueva Evaluación y Categorización). En julio de 2008, la Asamblea Nacional del Ecuador expidió el Mandato Constituyente $\mathrm{N}^{\circ} 14$ cuyo objetivo principal fue encargar al "CONEA la elaboración de una evaluación del desempeño institucional de todas el Instituciones de Educación Superior (IES) del Ecuador con la finalidad de depurar el sistema" (Rojas, 2011, p. 61). Los resultados de los informes del Mandato $\mathrm{N}^{\circ} 14,(2009)$ en cuanto al rol de las universidades en la formación de investigadores evidencian que:

La función de investigación es la más débil del Sistema de Educación Superior (SES), presentándose como grave en las universidades particulares. De esta manera, surge entonces la necesidad de incentivar la formación de sus docentes a nivel de $\mathrm{PhD}$ en pos de lograr un mejoramiento en la calidad de la educación superior (Rojas, 2011, p. 61).

Los resultados expuestos del informe dieron paso a que el CACES pueda "generar reflexiones y aprendizajes institucionales para mejorar los procesos a su cargo y responder a las necesidades actuales SES del país" (CACES, 2019, p.2), estableciendo un equilibrio entre la docencia, la investigación, producción científica e innovación y la vinculación con la sociedad.

2.2.2. La Secretaría de Educación Superior, Ciencia, Tecnología e Innovación (SENESCYT) La Secretaría de Educación Superior, Ciencia, Tecnología e Innovación (SENESCYT) es "la entidad del Gobierno ecuatoriano que ejerce la rectoría de la política pública en educación superior, ciencia, tecnología e innovación" (SENESCYT, 2019, secc. La Secretaría). De igual manera, es la encargada de regir la "política pública en los ámbitos de Ciencia, Tecnología, Innovación y Saberes Ancestrales; coordina y articula las acciones entre los sectores

Licencia Creative Commons Atribución 4.0 Internacional (CC BY 4.0)

Revista Cátedra, 3(2), pp. 126-149, mayo-agosto 2020. e-ISSN: 2631-2875

https://doi.org/10.29166/10.29166/catedra.v3i2.2183 
académico, de investigación, productivos público y privado" (SENESCYT, 2019, secc. La Secretaría). Dentro de esta institución se encuentra la Subsecretaría de Ciencia, Tecnología e Innovación en la cual se encuentran las subsecretarías de Investigación Científica e Innovación y Transparencia de Tecnología.

\subsubsection{Subsecretaría de Investigación Científica}

La Subsecretaría de Investigación Científica es la encargada de gestionar estratégicamente la política pública de investigación científica del país. En la página web de la Subsecretaría de Investigación Científica se encuentran atribuciones y responsabilidades de la misma y que respecto a la producción científica se destacan los siguientes literales:

b) Asesorar al Subsecretario/a General de Ciencia, Tecnología e Innovación, en la definición de actividades que promueven e impulsen la investigación científica;

c) Ejercer la rectoría de política pública de investigación científica

e) Aprobar, evaluar y financiar los planes, programas y proyectos de investigación científica de esta Secretaría; f) Validar y avalar los proyectos de investigación propuestos por los actores del Sistema Nacional de Ciencia, Tecnología, Innovación y saberes ancestrales;

g) Acreditar investigadores e instituciones de investigación para el desarrollo de actividades científicas en el país; h) Promover y articular redes de investigación científica; i) Articular a los actores del Sistema Nacional de Ciencia, Tecnología, Innovación y Saberes Ancestrales para la generación de investigación científica (secc. Subsecretaría de Investigación Científica).

El conjunto de literales destacados anteriormente, todos se relacionan con el fin de promover la investigación científica en las IES. Por ejemplo, el asesoramiento al Subsecretario para promover la investigación científica, la aprobación de proyectos investigativos son algunos de los aspectos relevantes que la SENESCYT desarrolla para el desarrollo la investigación científica dentro de las IES del país.

\subsubsection{Subsecretaría de Innovación y Transferencia de Tecnología}

Dentro de la esta Subsecretaría se encuentran determinadas atribuciones y responsabilidades que deberá cumplir el subsecretario General de Ciencia, Tecnología e Innovación. La relacionada con la producción científica del país es la planteada en el literal i) Dar seguimiento a la producción científica, a los programas y proyectos de investigación financiados por la Secretaría.

Estas atribuciones y responsabilidades de cada Subsecretaría perteneciente a la SENESCYT surgen a partir de lo estipulado en la LOES , donde en el Capítulo 3, COORDINACIÓN DEL SISTEMA DE EDUCACIÓN SUPERIOR CON LA FUNCIÓN EJECUTIVA, Art 182, literal g, estable que "desde el gobierno nacional, se deben crear políticas de investigación científica y tecnológica de acuerdo con las necesidades del desarrollo del país y crear los incentivos para que las universidades y escuelas politécnicas puedan desarrollarlas, sin menoscabo de sus políticas internas" (LOES, 2010a, p. 30).

La organización de esta entidad pública ha permitido un mejor desarrollo y cumplimiento de las funciones que ésta tiene en cuanto la rectoría de las políticas públicas relacionadas a la educación superior. De esta manera, el marco legal que rige al SES se complementa cumpliendo lo estipulado en la constitución de la república. En cuanto a los organismos que deben regir el Sistema de Educación Superior.

\section{Licencia Creative Commons Atribución 4.0 Internacional (CC BY 4.0)}

Revista Cátedra, 3(2), pp. 126-149, mayo-agosto 2020. e-ISSN: 2631-2875

https://doi.org/10.29166/10.29166/catedra.v3i2.2183 


\subsubsection{El Consejo de Educación Superior (CES)}

El Consejo de Educación Superior o también conocido como CES es uno de los organismos que rigen el Sistema de Educación Superior que tiene "como misión la planificación, regulación y coordinación interna del Sistema de Educación Superior del Ecuador, y la relación entre sus distintos actores con la Función Ejecutiva y la sociedad ecuatoriana" (Consejo de Educación Superior, 2012, sección institución). Se encarga de dirigir las universidades ecuatorianas mediante una serie de normativas.

Esta institución aprobó en el 2017 un reglamento de Régimen Académico encargado de regular los títulos y grados académicos y todo lo relacionado a ello. En uno de los títulos se establecen un sin número de leyes en torno a la actividad de investigación. Por ejemplo, se expresa en el artículo 72:

Investigación para el aprendizaje. - La organización de los aprendizajes en cada nivel de formación de la educación superior se sustentará en el proceso de investigación correspondiente y propenderá al desarrollo de conocimientos y actitudes para la innovación científica, tecnológica, humanística y artística, conforme a lo siguiente:

1. Investigación en educación técnica superior, tecnológica superior y sus equivalentes.- Se desarrollará en el campo formativo de creación, adaptación e innovación tecnológica, mediante el dominio de técnicas investigativas de carácter exploratorio. Las carreras artísticas deberán incorporar la investigación sobre tecnologías, modelos y actividades de producción artística.

2. Investigación en educación superior de grado. - Se desarrollará en el marco del campo formativo de la epistemología y la metodología de investigación de una profesión, mediante el desarrollo de proyectos de investigación de carácter exploratorio y descriptivo (Reglamento de Régimen Académico, 2017, p.31).

Es decir, las universidades, carreras y docentes de cada una de ellas tendrán la responsabilidad de añadir a las mallas curriculares la asignatura de investigación. De esta forma se busca dar herramientas sobre investigación a los futuros profesionales. Además, se trata de dar cumplimiento a la ley que establece que las IES deben generar ciencia. Los conocimientos que los estudiantes reciban sobre la elaboración de proyectos investigativos, tipos de investigaciones, metodologías, técnicas, instrumentos entre otros ayudará a que contribuyan a elevar el número de publicaciones dentro del país. Por consiguiente, se evita que la tarea investigativa sea realizada de forma superficial y con otros fines como el de acumular documentos en vez de generar conocimientos.

\subsection{Reforma Universitaria en el Ecuador}

La llegada a la Presidencia de la República del Ecuador del Economista Rafael Correa Delgado en el 2007 marcó un antes y un después en el Sistema de Educación Superior. En los años anteriores al 2007 "la investigación no formaba parte de los programas de estudio, las pocas que se realizaban respondían a inquietudes científicas de profesores y se convertían, generalmente, en tesis de grado o de doctorado" (Rivera-García, et al., 2017, p. 115). La realidad del país en cuanto a Investigación era totalmente deficiente y era necesario cambiarla. El modelo de Educación Superior en el Ecuador toma un giro en el año 2008 con la nueva Constitución de la República del Ecuador. En el Título VII, artículo 350 señala que:

El sistema de educación superior tiene como finalidad la formación académica y profesional de los estudiantes con una visión que nace desde

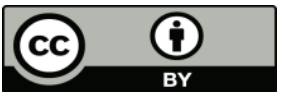

Licencia Creative Commons Atribución 4.0 Internacional (CC BY 4.0)

Revista Cátedra, 3(2), pp. 126-149, mayo-agosto 2020. e-ISSN: 2631-2875 https://doi.org/10.29166/10.29166/catedra.v3i2.2183 
las ciencias y las humanidades, de manera que se basa en la investigación científica y tecnológica, la innovación, promoción y difusión de los saberes ancestrales y las culturas. En función de dar solución a los problemas del país, en relación con los objetivos del régimen de desarrollo (p. 162).

Esta disposición promueve y exige que la Educación Superior sea vinculada con la investigación científica y tecnológica del país. Para lograrlo fue necesario la implementación de una nueva Ley de Educación Superior, misma que entró en vigencia en octubre del 2010. Esta nueva LOES en el artículo 13 determina las funciones del Sistema de Educación Superior, entre ellas se destacan los literales:

b) Promover la creación, desarrollo, transmisión y difusión de la ciencia,

la técnica, la tecnología y la cultura;

d) Fortalecer el ejercicio y desarrollo de la docencia y la investigación científica en todos los niveles y modalidades del sistema (LOES, 2010, p.

6).

Con la nueva visión del Sistema de Educación Superior planteados en la Constitución de la República del 2008 y Ley Orgánica de Educación Superior del 2010 fue necesario plantear un nuevo marco legal que rija la educación superior en el Ecuador. Este marco legal estaría conformado por la Secretaría Nacional de Educación Superior, Ciencia, Tecnología e Innovación (SENESCYT); el Consejo de Educación Superior (CES) y el Consejo de Aseguramiento de la Calidad de la Educación Superior (CACES). Este último estaría a cargo de la evaluación y categorización de las Universidades del Ecuador.

\section{Trabajos relacionados}

En el año 2013, un seminario realizado en la Escuela Politécnica Nacional presentó un análisis bibliométrico comparativo del Ecuador a nivel mundial y de algunas universidades del país. Los resultados arrojan que el Ecuador hasta el 2012 ha indexado con Scopus 3649 y en la Web of Knowledge 3573 artículos científicos, por otra parte, las Instituciones de Educación Superior más prolíficas se encuentran la Universidad San Francisco de Quito en primer lugar, la Pontificia Universidad Católica del Ecuador en segundo lugar y la Escuela Politécnica Nacional en tercer lugar. El seminarista concluye que el Ecuador necesita un Plan Nacional de Ciencia y Tecnología que motive a la producción de proyectos e investigaciones para lograr un cambio en la matriz productiva (Bruque, 2013).

En otra investigación de 2015, se realizó una comparación de la Universidad San Francisco de Quito con otras universidades del Ecuador. Los resultados otorgados por Sir Iberoamérica 2015 se pudo observar que la USFQ es la universidad con mayor número de producción científica convirtiéndose en una de las 200 instituciones de educación superior más productivas de Iberoamérica. Finalmente, el autor llegó a la conclusión de que la USFQ proporciona el $74,14 \%$ de las publicaciones de categoría alta y que el 82,89\% las realiza en con colaboración con instituciones internacionales lo que revela el prestigio que está adquiriendo la USFQ a nivel nacional y mundial (DFHC,2015).

En el 2015, se desarrolló un estudio sobre la investigación científica en las universidades ecuatorianas, en el cual el autor aborda uno de los principales problemas de los estudiantes a nivel nacional y sobre todo de la educación superior. Los resultados de esta investigación se relacionan a la Universidad ecuatoriana con el gobierno y la inversión que éste hace para las Instituciones de Educación Superior. Por otro lado, se plantea que la producción académica y científica está rodeada de prejuicios y se ve limitada por los mismos. El estudio

Licencia Creative Commons Atribución 4.0 Internacional (CC BY 4.0)

Revista Cátedra, 3(2), pp. 126-149, mayo-agosto 2020. e-ISSN: 2631-2875

https://doi.org/10.29166/10.29166/catedra.v3i2.2183 
concluyó que se debe cambiar verdaderamente la educación superior alejándola del modelo universitario norteamericano de los años sesenta (Ayala, 2015).

En el año 2016, una investigación realizó un estudio sobre la perspectiva de la difusión de conocimientos del Ecuador a nivel nacional, regional y mundial. En los resultados se determina que Ecuador ocupa el puesto 82 a nivel mundial con 3662 documentos científicos, el puesto 13 a nivel de Latinoamérica. Por último, se realiza una comparación entre el ranking de universidades ecuatorianas con mayor número de publicaciones indexadas en el periodo de 2009-2013. La lista de cinco Instituciones de Educación Superior con mayor número de textos científicos se menciona en el siguiente orden:

1. Universidad San Francisco de Quito con 422 artículos científicos en Scopus

2. Pontificia Universidad Católica del Ecuador con 319 artículos científicos en Scopus

3. Escuela Politécnica Nacional del Ecuador con 152 artículos científicos en Scopus

4. Universidad Técnica Particular de Loja con 143 artículos científicos en Scopus

5. Escuela Politécnica del Litoral con 137 artículos científicos en Scopus

Finalmente, los autores concluyen que las tendencias a nivel nacional, regional y mundial demuestran la importancia que las universidades han ido atribuyéndole a la investigación y la divulgación de resultados (Méndez, et al., 2016).

En un estudio en el año 2016, se realizó un análisis del nivel de producción científica de los docentes de Nivelación de la Universidad de Guayaquil hasta el primer semestre del mismo año. Los resultados permitieron corroborar que 42 docentes, 28 hombres y 14 mujeres, han publicado artículos científicos es decir el 17,5 \% de la planta de educadores de la Universidad. Debido al problema encontrado, los investigadores concluyen en que es necesario sistematizar el conocimiento para lograr el desarrollo de competencias pluridisciplinarias y pensamiento multidimensional de los docentes con el fin de que generen innovaciones y nuevas investigaciones (Reiban-Barrera y Vera-Cedeño, 2016).

Un estudio presentado en el año 2017 realizó una comparación sobre el número de artículos científicos entre la Universidad Técnica Particular de Loja, la Universidad Nacional de Loja y la Universidad Politécnica del Litoral. En los resultados se determina que la UTPL ha publicado 544 artículos desde el 2012, la UNL ha publicado 102 textos científicos mientras que la Escuela Politécnica del Litoral ha publicado 531 desde el año 2011. Por consiguiente, el autor de esta investigación concluye que se evidencia notablemente un incremento de producción científica debido a la inversión que el Gobierno efectúo en Educación Superior (Añazco, 2017).

La investigación presentada en el 2017 por la Revista Cuba de Educación Superior analiza la investigación científica en las universidades ecuatorianas como prioridad del sistema educativo vigente. Los resultados de esta investigación arrojaron que en las universidades ecuatorianas hasta los años setenta carecían del componente investigativo y que a pesar de que el Estado ecuatoriano a partir de la década de los años ochenta promovió a través de varias organizaciones la investigación científica jamás las universidades ecuatorianas lograron cumplir con las expectativas señaladas gracias a la ruptura que existía entre la docencia y la investigación. Por lo tanto, concluye que a pesar de los cambios generados en la investigación científica dentro de las universidades, el desarrollo general no es suficiente. A demás no existe una comprensión total por parte de todos los miembros de las Instituciones de Educación Superior sobre a las acciones a tomar para incrementar la producción académica dentro de sus aulas (Rivera-García et al., 2017).

En el año 2018, se presenta una investigación relacionada con la producción científica de la Universidad Ecuatoriana con la finalidad de conocer cuál es la producción académica Licencia Creative Commons Atribución 4.0 Internacional (CC BY 4.0)

Revista Cátedra, 3(2), pp. 126-149, mayo-agosto 2020. e-ISSN: 2631-2875 https://doi.org/10.29166/10.29166/catedra.v3i2.2183 
científica en las universidades del Ecuador y todo lo relacionado a la capacitación de los trabajos de investigación en las Instituciones de Educación Superior. Los resultados de esta reflejan que la producción científica incrementa cuando se tiene profesionales debidamente preparados, también que la investigación ha logrado solucionar problemas en el ámbito de la ciencia y mejorando el desempeño de los profesionales relacionados con la producción científica. Por lo tanto, se concluyó que el incremento de la producción científica en los últimos años fue gracias a la intervención de algunas entidades relacionadas con la Educación Superior (Cabrera, et al., 2018).

En el 2019, se realiza un análisis de la Producción Científica de las Universidades de las Zonas 3,5 y 8 del Ecuador. En este estudio se comparó la producción científica de la Universidad Estatal de Milagro, Universidad de Guayaquil, Universidad Nacional del Chimborazo y la Universidad Estatal de Bolívar. Los resultados de esta comparación fueron que, en el año 2015, la Universidad Nacional del Chimborazo fue la que más publicaciones científicas tuvo con un total de 84 documentos y para el 2017 mantuvo el primer lugar con un total de 190 publicaciones. Este estudio concluyó que la producción científica había incrementado notablemente para el año 2017 en todas las universidades de las Zonas 3, 5 y 8. del Ecuador (Pacheco, et al., 2019).

\section{Metodología}

A continuación, se describen los pasos utilizados en esta investigación:

1. Selección del tipo de investigación de la metodología: Esta investigación fue exploratoria por lo que para poder realizarla fue necesario una revisión exhaustiva de la literatura relacionada con el tema. El presente estudio manejó una metodología cualitativa inductiva-deductiva basado en el empleo de la hermenéutica para la interpretación de datos obtenidos de la revisión de la literatura.

2. Selección de una muestra representativa de Instituciones de Educación Superior: se seleccionaron 10 universidades ecuatorianas tomando en cuenta el listado proporcionado por Diario El Comercio sobre las 15 mejores universidades ecuatorianas según el QS World University Rankings 2020. Estas instituciones de Educación Superior fueron calificadas con base 8 criterios. Esos criterios son: la reputación académica de la institución, la reputación del empleador, la relación estudiante- facultad, el staff $\mathrm{PhD}$, la red de investigación internacional, las citaciones por investigaciones, las investigaciones por facultad y el impacto en Internet (El Comercio,2020).

Las universidades seleccionadas fueron la Universidad San Francisco de Quito, Escuela Superior Politécnica del Litoral, Pontificia Universidad Católica del Ecuador, Escuela Politécnica Nacional, Universidad Central del Ecuador, Universidad de Cuenca, Universidad de las Fuerzas Armadas, Universidad Técnica Particular de Loja, Universidad Espíritu Santo, Universidad de las Américas.

3. Selección de fuentes bibliográficas: para poder conocer el número de producciones científicas que han tenido las 10 universidades fue necesario buscar fuentes de datos confiables. Después de haber revisado varias fuentes bibliográficas se llegó al acuerdo que los datos serían obtenidos de las ediciones 2010, 2012,2015 y 2017 del Ranking Iberoamericano SIR. Es necesario, recalcar que cada edición emitida por el Scimago institutions ranking presenta informes agrupados en quintiles. Por ello, los períodos tomados en cuenta para este estudio son: 20032008, 2006-2010, 2009-2013 y 2013-2017. Estos informes fueron tomados gracias a la aproximada secuenciación que existe entre los años 2003-2017. Otros informes 
no permitían cumplir con el propósito de este estudio ya que omitían años importantes e impedía la adecuada secuenciación de datos.

4. Comparación de universidades ecuatorianas: para contrastar datos entre universidades se decidió elaborar cuadros y gráficas que ayudaron a ver el número de publicaciones por períodos de cinco años. De esta manera se pudo observar si existía una mejora o un descenso en el número de artículos científicos producidos. También, se buscó advertir algún cambio en el puesto que la Instituciones de Educación Superior ocupaba a nivel nacional, iberoamericano y latinoamericano. Por último, para establecer el posible desarrollo entre las universidades se decidió comparar los períodos 2003-2008 y 2006-2010.

5. Resultados

5.1. Producciones científicas indexadas a Scopus por quinquenios

El Scimago institutions rankings (SIR) es la fuente bibliográfica de la que se ha podido extraer los datos sobre las publicaciones científicas elaboradas por universidades ecuatorianas en los períodos de 2003-2008, 2006-2010, 2009-2013 y 2013-2017. SIR (2010) "se presenta como una herramienta de análisis y evaluación de la actividad investigadora de las Instituciones de Educación Superior en Iberoamérica" (p.1). Es decir, los informes presentados permiten conocer la situación actual de un país y de las universidades con respecto a la creación de artículos científicos que ayuden al desarrollo de la sociedad. Los datos presentados por períodos de cinco años pueden servir como referentes para la planificación o aplicación de posibles soluciones en pro del aumento de producción científica en un país.

\begin{tabular}{|c|c|c|c|c|}
\hline Universidades & $\begin{array}{l}2003- \\
2008\end{array}$ & $\begin{array}{l}2006- \\
2010\end{array}$ & $\begin{array}{l}2009- \\
2013\end{array}$ & $\begin{array}{l}2013- \\
2017\end{array}$ \\
\hline Universidad San Francisco de Quito & 200 & 318 & 422 & 836 \\
\hline $\begin{array}{l}\text { Escuela Superior Politécnica del } \\
\text { Litoral }\end{array}$ & 3 & 71 & 137 & 730 \\
\hline $\begin{array}{l}\text { Pontificia Universidad Católica del } \\
\text { Ecuador }\end{array}$ & 118 & 221 & 319 & 571 \\
\hline Escuela Politécnica Nacional & 113 & 133 & 152 & 691 \\
\hline Universidad Central del Ecuador & 50 & 59 & 103 & 348 \\
\hline Universidad de Cuenca & 32 & 51 & 91 & 503 \\
\hline Universidad de las Fuerzas Armadas & 0 & 19 & 53 & 884 \\
\hline $\begin{array}{l}\text { Universidad Técnica Particular de } \\
\text { Loja }\end{array}$ & 17 & 53 & 143 & 732 \\
\hline Universidad Espíritu Santo & 0 & 0 & 34 & 222 \\
\hline Universidad de las Américas & 0 & 8 & 90 & 245 \\
\hline
\end{tabular}

Cuadro 1. Número de publicaciones realizadas por cada una de las diez Instituciones de Educación Superior más representativas del Ecuador indexadas a Scopus dentro de los períodos 2003-2008, 2006-2010, 20092013 y 2013-2017. Adaptado de: (SIR,2010,2012,2015 y 2019)

El primer período que se toma en cuenta es desde el año 2003 al 2008. Durante este transcurso de tiempo el SIR ha recopilado el número de artículos científicos que han escrito cada una de las universidades ecuatorianas indexadas a Scopus. Para la elaboración del informe de resultados "se han analizado las publicaciones científicas incluidas en el índice

Licencia Creative Commons Atribución 4.0 Internacional (CC BY 4.0)

Revista Cátedra, 3(2), pp. 126-149, mayo-agosto 2020. e-ISSN: 2631-2875

https://doi.org/10.29166/10.29166/catedra.v3i2.2183 
de citas Scopus producido por ESLEIVER" (SIR, 2010). Es relevante recordar que los datos presentados en el ranking corresponden a toda Iberoamérica y Latinoamérica por lo que para realizar la siguiente figura fue necesario extraer solo lo referente a las Instituciones de Educación Superior del Ecuador. La figura 1 muestra a las mejores diez universidades del Ecuador y el número de publicaciones que tuvieron dentro de estos años.

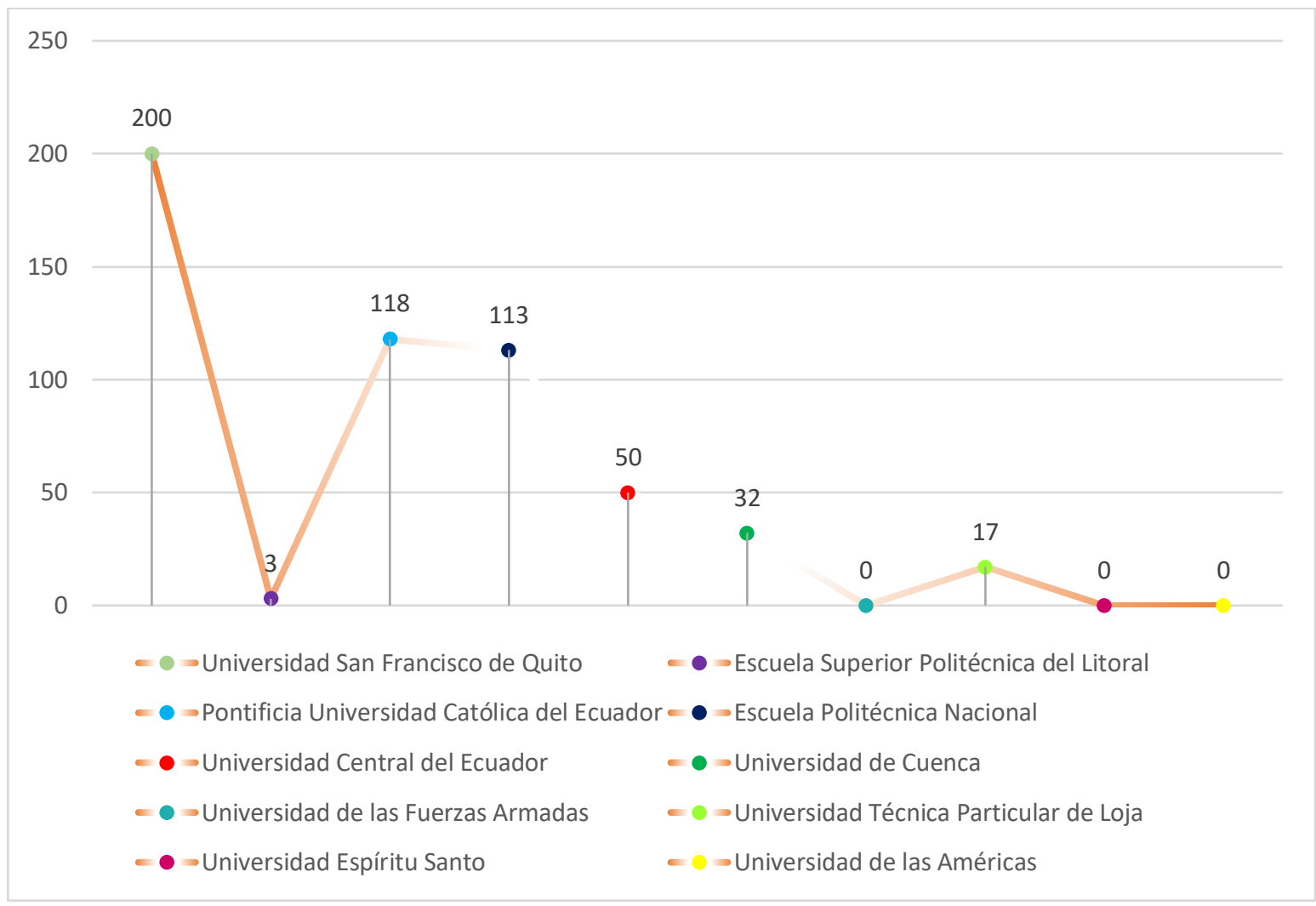

Figura 1. Número de publicaciones en realizadas por cada una de las diez Instituciones de Educación Superior más representativas del Ecuador indexadas a Scopus dentro del período 2003-2008.

Después de observar detenidamente la figura 1 se puede contrastar que las tres universidades con más publicaciones en el período de 2003 a 2008 fueron la Universidad San Francisco de Quito (USFQ), la Pontificia Universidad Católica del Ecuador (PUCE) y la Escuela Politécnica Nacional (EPN). La USFQ ocupó el primer lugar con 222 publicaciones, posteriormente la PUCE obtuvo el segundo lugar con 118 publicaciones y el tercer lugar fue para la EPN con 113 publicaciones. En cuanto a las otras, la Universidad Central del Ecuador cuenta con 50 artículos científicos, la Universidad de Cuenca realizó 32 artículos científicos, la Universidad Técnica Particular de Loja con 17 artículos científicos y la Escuela Politécnica del Litoral con apenas 3 publicaciones. Mientras que las Universidades con nula producción científica fueron la Universidad de las Fuerzas Armadas, la Universidad Espíritu Santo y la Universidad de las Fuerzas Armadas.

El segundo período tomado en cuenta es el quinquenio del 2006 al 2010. Durante este transcurso de tiempo el SIR recopiló el número de artículos científicos que escribieron cada una de las universidades ecuatorianas. Para la elaboración del informe de resultados "se ha analizado toda la producción científica presente en la base de datos Scopus, elaborada por Elsevier, 2016 en el periodo 2006-2010 y se ha asociado cada publicación y cada cita encontrada a la institución o instituciones correspondientes" (SIR, 2012). Es de importancia indicar que los datos presentados en el ranking corresponden a toda Iberoamérica y 
Latinoamérica razón por la cual para elaborar la siguiente figura fue necesario extraer sólo la información referente a las universidades del Ecuador. La figura 2 muestra a las mejores diez universidades del Ecuador y el número de publicaciones que tuvieron dentro de este período.

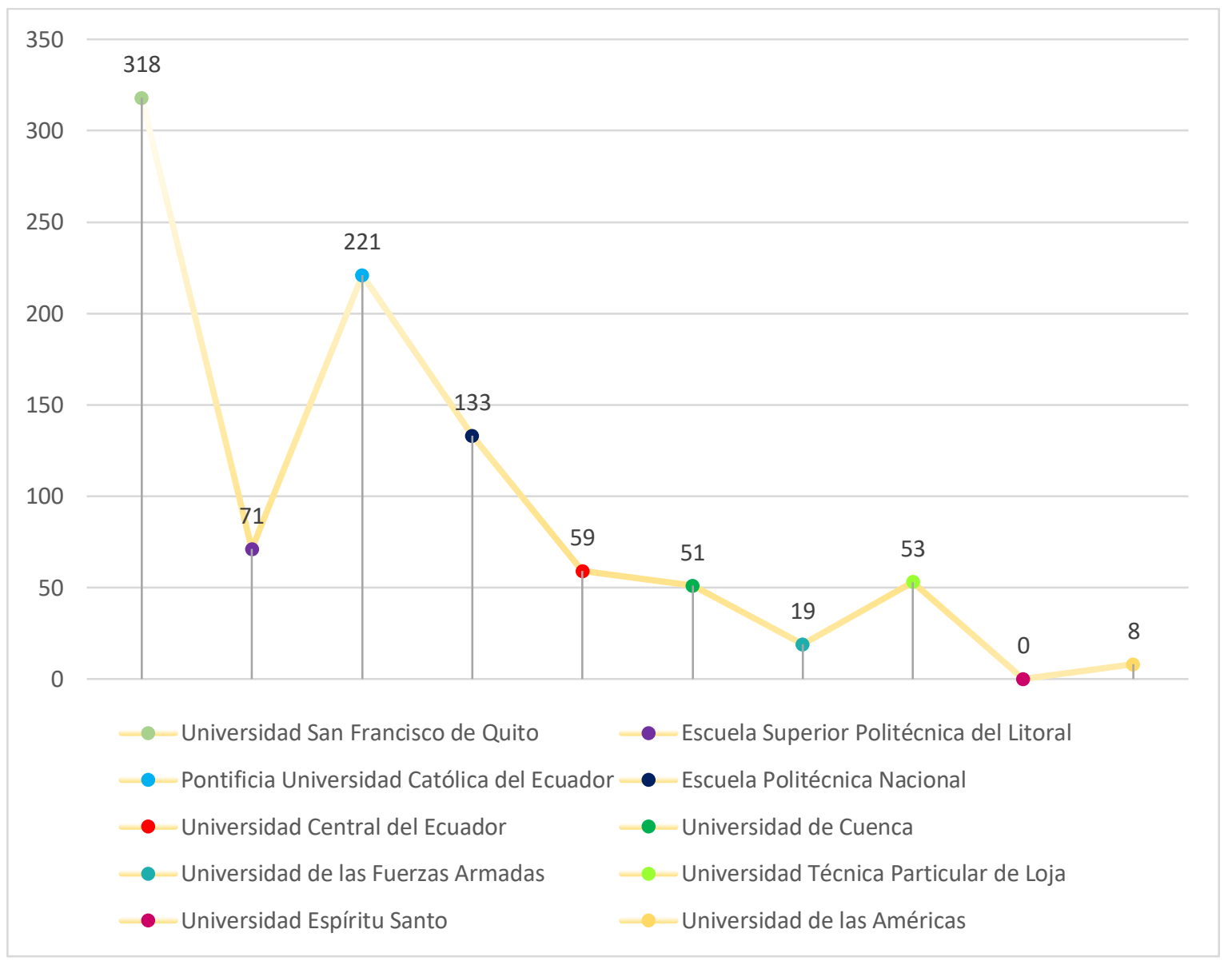

Figura 2. Número de publicaciones realizadas por cada una de las diez Instituciones de Educación Superior más representativas del Ecuador indexadas a Scopus dentro del período 2006-2010.

En la figura anterior se puede contrastar que las tres universidades con más publicaciones durante los años 2006-2010 fueron la Universidad San Francisco de Quito ocupando el primer lugar con un total de 318 publicaciones, seguida de la Pontificia Universidad Católica del Ecuador con un total de 221 publicaciones y en tercer lugar con un total de 133 publicaciones se encontró la Escuela Politécnica Nacional. Por otra parte, las Instituciones de Educación Superior con menos producción científica fueron la Universidad de las América con un número de publicaciones igual a 8 y la Universidad Espíritu Santo con una inexistente producción científica durante este período.

Los años 2009-2013 conforman el tercer período tomado en cuenta para el desarrollo de esta investigación. Durante este transcurso de tiempo el SIR recopiló el número de artículos científicos que se han escrito en cada una de las universidades ecuatorianas. Es de importancia indicar que los datos presentados en el ranking corresponden a toda Iberoamérica y Latinoamérica razón por la cual para elaborar la siguiente figura fue necesario extraer sólo la información referente a las Instituciones de Educación Superior del Ecuador. La figura 3 muestra a las mejores diez universidades del Ecuador y el número de publicaciones que tuvieron dentro de este período.

\section{Licencia Creative Commons Atribución 4.0 Internacional (CC BY 4.0)}

Revista Cátedra, 3(2), pp. 126-149, mayo-agosto 2020. e-ISSN: 2631-2875

https://doi.org/10.29166/10.29166/catedra.v3i2.2183 


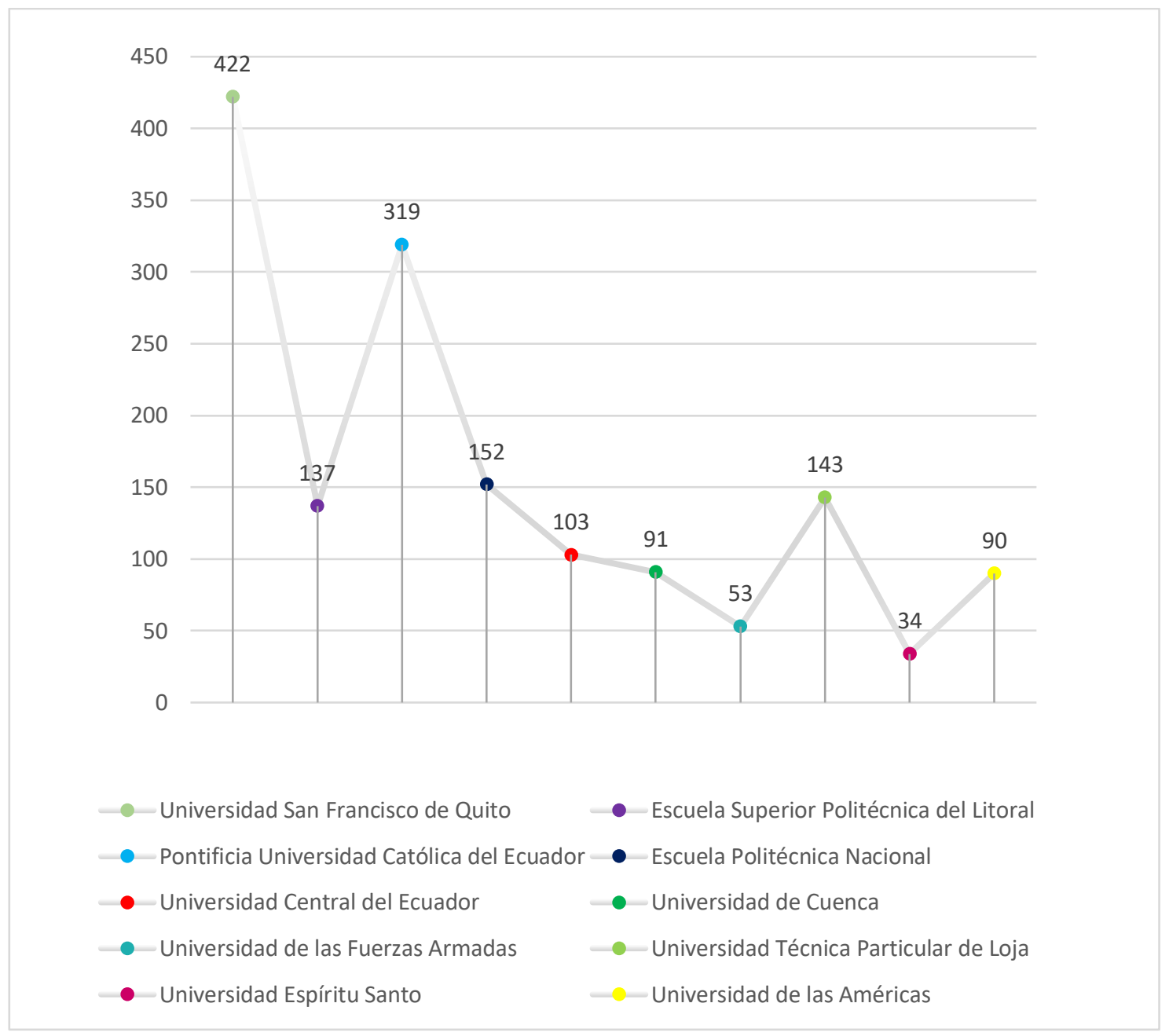

Figura 3. Número de publicaciones realizadas por cada una de las diez Instituciones de Educación Superior más representativas del Ecuador indexadas a Scopus del período 2009-2013.

En la figura 3 se puede contrastar que las universidades con más publicaciones durante los años 2009-2013 fueron la Universidad San Francisco de Quito ocupando el primer lugar con un total de 422 publicaciones, seguida de la Pontificia Universidad Católica del Ecuador con un total de 319 publicaciones y en tercer lugar con un total de 152 publicaciones se ubicó la Escuela Politécnica Nacional. Por otra parte, las Instituciones de Educación Superior con menos producción científica fueron la Universidad Espíritu Santo con un total de 34 publicaciones y la Universidad de las Fuerzas Armadas con un número de publicaciones igual a 53 .

El último período tomado en cuenta para este estudio fue el quinquenio del 2013 al 2017. Durante este tiempo el SIR analiza "la capacidad institucional de generar productos científicos y difundirlos a través de canales reconocidos de comunicación científica" (SIR, 2019, p. 24). Es de importancia indicar que los datos presentados en el ranking corresponden a toda Iberoamérica y Latinoamérica razón por la cual para elaborar la siguiente figura fue necesario extraer sólo la información referente a las Instituciones de Educación Superior del Ecuador. La figura 4 muestra a las mejores diez universidades del Ecuador con el número correspondiente de publicaciones científicas durante este último período. 


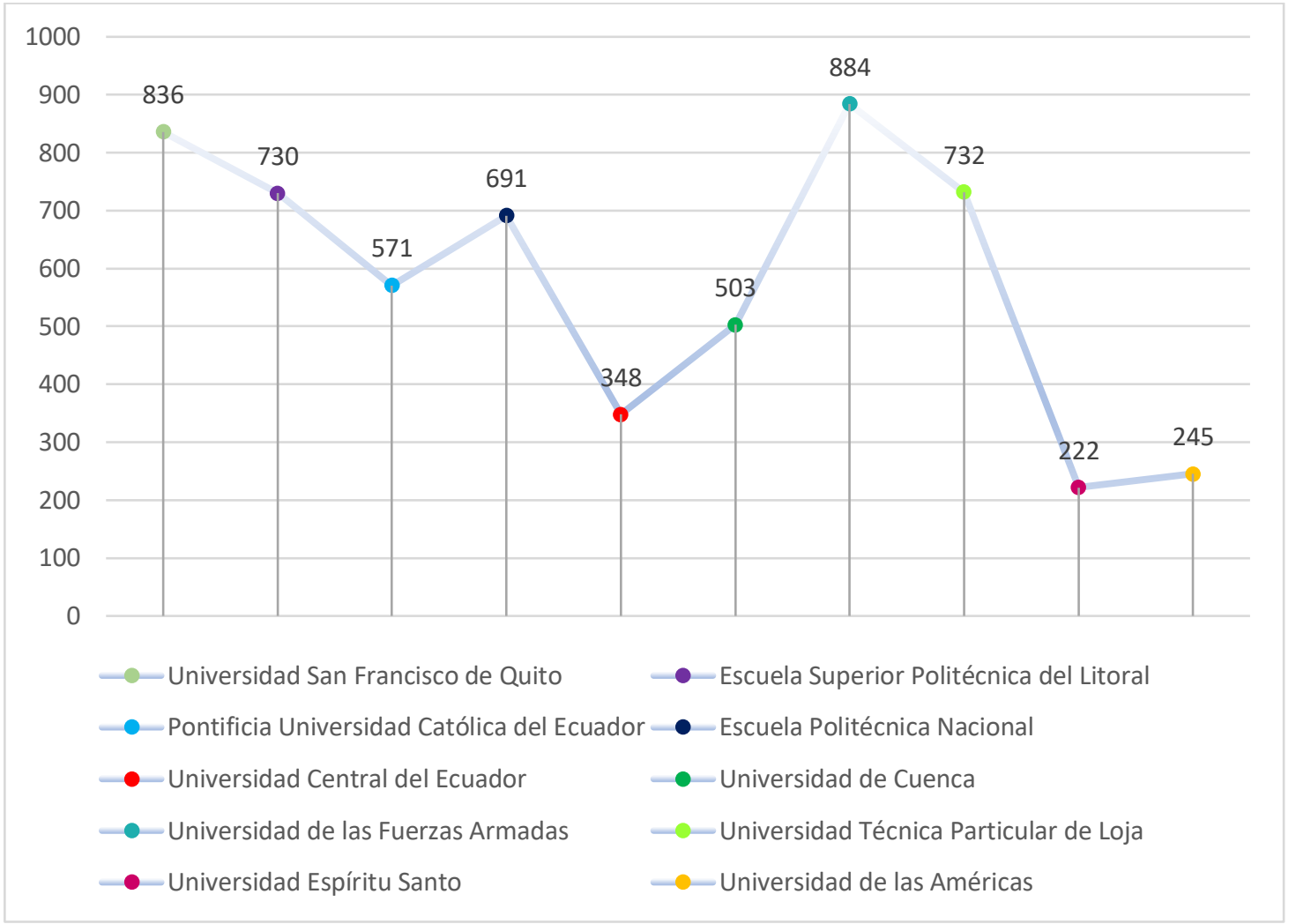

Figura 4. Número de publicaciones realizadas por cada una de las diez Instituciones de Educación Superior más representativas del Ecuador indexadas a Scopus dentro del período 2013-2017.

En la figura 4 se puede contrastar que las universidades con más publicaciones durante los años 2013-2017 fueron: la Universidad de las Fuerzas Armadas con un total de 884 publicaciones, en segundo lugar, la Universidad San Francisco de Quito con 836 publicaciones y en tercer lugar la Universidad Técnica Particular de Loja con un total de 732 publicaciones. Por otra parte, también se contrasta que las Universidades con menos producción científica en este período fueron la Universidad de las América con un número de publicaciones igual a 245 y la Universidad Espíritu Santo con un total de 222 publicaciones en Scopus.

\subsection{Universidades ecuatorianas según su puesto a nivel nacional, iberoamericano y latinoamericano por quinquenio}

SCImago Research Group desde el año 2009 publica dos tipos de informes: SIR world y SIR Iber. Este último informe "muestra la actividad científica exclusivamente de Andorra, España, Portugal, y los países de Latinoamérica" (SIR, 2019, p. 5). Para la medición de la producción científica el SIR toma en cuenta tres aspectos: investigación, innovación e impacto social. Dentro del aspecto de investigación se pretende medir "la capacidad institucional de generar productos científicos y difundirlos a través de canales reconocidos de comunicación científica". (SIR, 2019, p. 24). Esta capacidad de producción científica que tiene cada Institución de Educación Superior le ha permitido posicionarse dentro del Ranking. En el cuadro 2 se muestran a las diez mejores Universidades del Ecuador durante los períodos 2003-2008, 2006-2010, 2009-2013 y 2013-2017 y el puesto que ocupan a nivel Iberoamericano, Latinoamericano y a nivel nacional.

\section{Licencia Creative Commons Atribución 4.0 Internacional (CC BY 4.0)}

Revista Cátedra, 3(2), pp. 126-149, mayo-agosto 2020. e-ISSN: 2631-2875 https://doi.org/10.29166/10.29166/catedra.v3i2.2183 


\begin{tabular}{|c|c|c|c|c|c|c|c|c|c|c|c|c|}
\hline Período & 200 & $3-20$ & & 20 & $5-2$ & & 20 & -20 & & 20 & $3-20$ & \\
\hline Universidades & IB & LA & $\mathrm{C}$ & IB & LA & $\mathrm{C}$ & IB & LA & $\mathrm{C}$ & IB & LA & $\mathrm{C}$ \\
\hline & $\mathrm{E}$ & $\mathrm{C}$ & 0 & $\mathrm{E}$ & $\mathrm{C}$ & 0 & $\mathrm{E}$ & $\mathrm{C}$ & 0 & $\mathrm{E}$ & $\mathrm{C}$ & 0 \\
\hline Universidad & 24 & 16 & 1 & 25 & 18 & 1 & 26 & 19 & 1 & 24 & 16 & 2 \\
\hline Francisco de Quito & 4 & 7 & & 8 & 1 & & 7 & 0 & & 0 & 4 & \\
\hline Escuela $\quad$ Superior & 55 & 44 & 1 & 49 & 39 & 4 & 40 & 31 & 5 & 25 & 18 & 4 \\
\hline Politécnica del Litoral & 6 & 6 & 1 & 3 & 4 & & 5 & 8 & & 8 & 0 & \\
\hline Pontificia Universidad & 29 & 20 & 2 & 30 & 22 & 2 & 30 & 22 & 2 & 29 & 20 & 6 \\
\hline Católica del Ecuador & 1 & 5 & & 5 & 4 & & 4 & 2 & & 1 & 7 & \\
\hline Escuela Politécnica & 29 & 21 & 3 & 39 & 30 & 3 & 39 & 30 & 3 & 26 & 18 & 5 \\
\hline Nacional & 7 & 1 & & 2 & 3 & & 4 & 7 & & 3 & 3 & \\
\hline Universidad Central del & 36 & 26 & 4 & 52 & 42 & 6 & 43 & 34 & 6 & 37 & 28 & 9 \\
\hline Ecuador & 2 & 9 & & 0 & 1 & & 9 & 3 & & 1 & 2 & \\
\hline Universidad de Cuenca & 39 & 30 & 6 & 55 & 44 & 9 & 43 & 35 & 7 & 30 & 22 & 7 \\
\hline & 9 & 0 & & 2 & 9 & & 9 & 2 & & 5 & 0 & \\
\hline Universidad de & 0 & 0 & 0 & 74 & 62 & 1 & 47 & 38 & 9 & 23 & 15 & 1 \\
\hline Fuerzas Armadas & & & & 6 & 8 & 2 & 6 & 8 & & 2 & 6 & \\
\hline Universidad Técnica & 45 & 35 & 1 & 54 & 44 & 7 & 40 & 31 & 4 & 25 & 17 & 3 \\
\hline Particular de Loja & 7 & 1 & 0 & 6 & 3 & & 0 & 3 & & 7 & 8 & \\
\hline Universidad Espíritu & 0 & 0 & 0 & 0 & 0 & 0 & 40 & 40 & 1 & 43 & 34 & 1 \\
\hline Santo & & & & & & & 5 & 6 & 1 & 4 & 2 & 1 \\
\hline Universidad & 0 & 0 & 0 & 93 & 80 & 1 & 50 & 41 & 1 & 42 & 32 & 1 \\
\hline Américas & & & & 8 & 8 & 7 & 4 & 5 & 4 & 1 & 9 & 0 \\
\hline
\end{tabular}

Cuadro 2. Puesto que ocupan cada una de las diez Instituciones de Educación Superior del Ecuador según su producción científica en Scopus a nivel iberoamericano (IBE), Latinoamericano y el Caribe (LAC) y dentro del país (CO). Adaptado de: (SIR,2010, 2012, 2015 y 2019).

\subsection{Producción científica antes y después de la Reforma de Educación Superior de 2008}

En el año 2008 en el mandato del Economista Rafael Correa Delgado, el país inicia una serie de cambios significativos entre ellos se pone en marcha la denominada "Revolución Educativa" y bajo el nombre de esta se propone una transformación radical en el Sistema de Educación Superior. Las universidades del Ecuador en cuanto a la investigación y producción científica antes del 2008 presentaban un desempeño deficiente. Rojas (2011) señala "la función de investigación es la más débil del Sistema de Educación Superior" (p. 61). Con el nuevo modelo educativo implementado en las universidades del Ecuador el componente de investigación y producción científica en las Instituciones de Educación Superior mejoraría notablemente. En la figura 5 se puede contrastar el incremento de la producción científica de las 10 mejores universidades del país antes y después de la reforma educativa de 2008. 


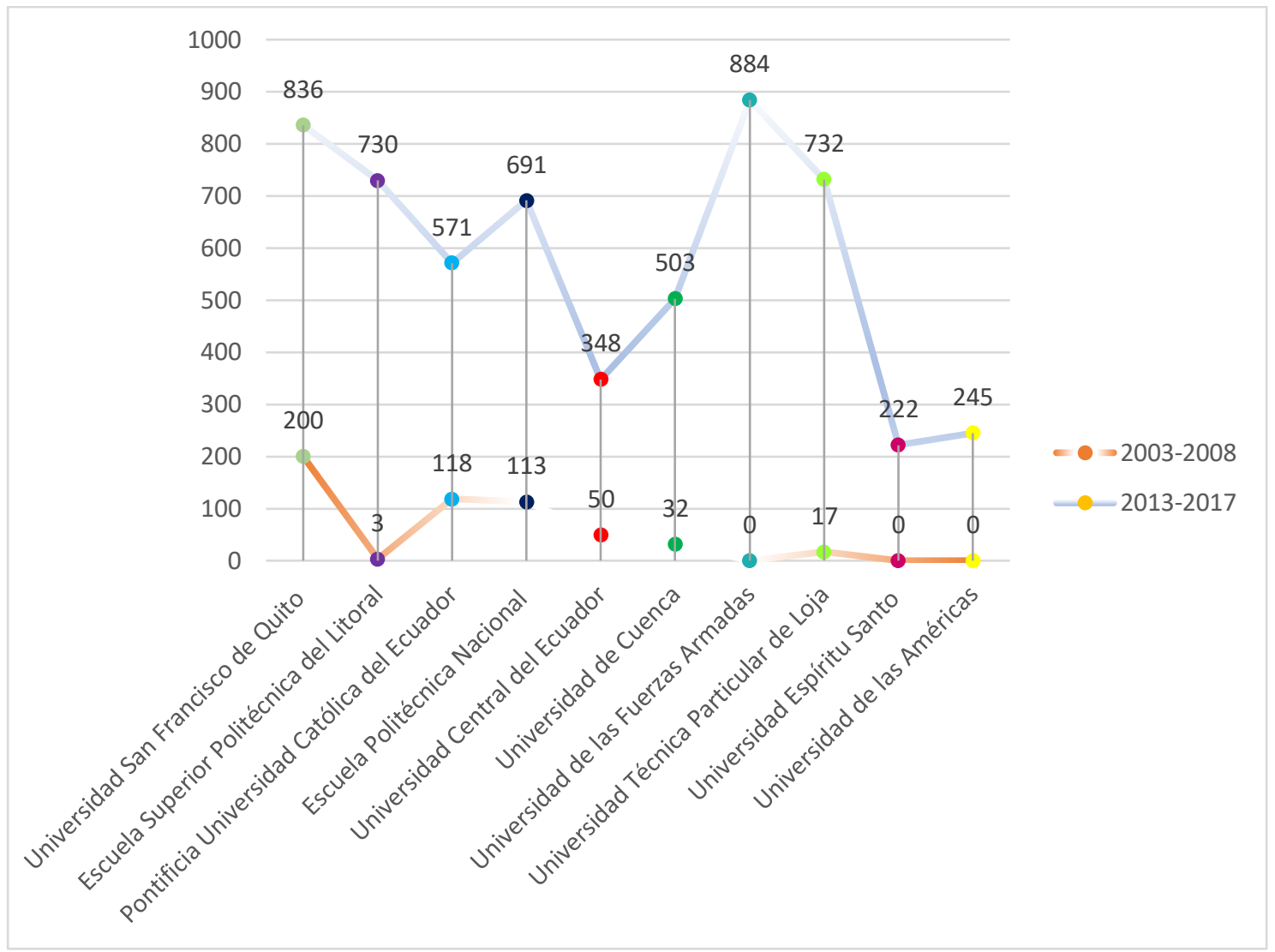

Figura 5. Comparación del número de publicaciones realizadas por cada una de las diez Instituciones de Educación Superior más representativas del Ecuador indexadas a Scopus entre el período 2003-2008 y el período 2013-2017

En la figura 5 se puede comparar el número de producción científica de las universidades más representativas del Ecuador antes y después del año 2008. Durante el período 20032008 las tres universidades con mayor producción científica fueron: Universidad San Francisco de Quito con 200 publicaciones, la Pontificia Universidad Católica del Ecuador con 118 publicaciones y la Escuela Politécnica Nacional con 113 publicaciones. El incremento de la producción científica es contrastado con los resultados del quinquenio 2013-2017, período en el cual estas universidades aumentan significativamente el número de publicaciones científicas. Siendo así, la Universidad San Francisco de Quito realiza 636 publicaciones más llegando a un total de 836 publicaciones, la Pontificia Universidad Católica del Ecuador alcanza las 571 publicaciones y la Escuela Politécnica Nacional llega a las 691 publicaciones.

Por otro lado, la comparación de las Universidades con menos producción científica durante el período 2003-2008 fueron la Universidad Espíritu Santo y la Universidad de las América, ambas con una inexistente producción científica en este período. Sin embargo, en el período 2013-2017 ambas universidades incrementan su producción científica con un total de 222 y 245 publicaciones en la base de datos Scopus respectivamente.

\section{Discusión de resultados}

En la figura 1 se presentó el número de publicaciones realizadas por cada una de las diez Instituciones de Educación Superior (IES) más representativas del Ecuador indexadas a Scopus dentro del período 2003-2008. Con base en estos resultados se aprecia que el 
número de publicaciones de estas universidades es bajo. Durante este período entre las diez IES hubo un total de 533 publicaciones de las cuales 431 publicaciones pertenecen a solo tres IES como son la Universidad San Francisco de Quito, la Pontificia Universidad Católica del Ecuador y la Escuela Politécnica Nacional. Por otra parte, la producción científica durante este período no fue significativa con respecto a las otras siete IES, como se observa en los datos presentados en la figura 1, las siete universidades restantes no alcanzaron las 100 publicaciones indexadas a Scopus.

En la figura 2 se presentó el número de publicaciones realizadas por cada una de las diez Instituciones de Educación Superior (IES) más representativas del Ecuador indexadas a Scopus dentro del período 2006-2010. Durante este quinquenio, en el año 2008, entró en vigencia la nueva reforma de educación superior con la cual se empieza a incrementar la producción científica en cada una de las IES. Esta afirmación es corroborada en la figura 2 pues todas las IES a excepción de la Universidad de Especialidades Espíritu Santo aumentan significativamente el número de publicaciones como es el caso de la Universidad San Francisco de Quito que en este período indexó a Scopus 318 publicaciones, es decir con respecto al quinquenio anterior incrementó un total de 118 publicaciones.

En la figura 3 se presentó el número de publicaciones realizadas por cada una de las diez Instituciones de Educación Superior (IES) más representativas del Ecuador indexadas a Scopus dentro del período 2009-2013. Con base en estos resultados se observa que el número de publicaciones de estas universidades incrementó considerablemente con respecto al quinquenio anterior. De las diez IES del Ecuador únicamente cuatro Universidades no alcanzaron las cien publicaciones indexadas a Scopus, sin embargo, se superaron considerablemente en comparación a períodos anteriores. Entre estas Instituciones se encuentran La Universidad de Cuenca, la Universidad de las Fuerzas Armadas, Universidad de Especialidades Espíritu Santo y Universidad de las Américas.

En la figura 4 se presentó el número de publicaciones realizadas por cada una de las diez Instituciones de Educación Superior (IES) más representativas del Ecuador indexadas a Scopus dentro del período 2013-2017. Con base en estos resultados se aprecia que ninguna de las diez IES tiene menos de 200 publicaciones indexadas a Scopus. De igual manera se evidencia que con un total de 884 publicaciones la Universidad de las Fuerzas ocupa el primer lugar durante este periodo, además se corrobora que la reforma de educación superior de 2008 influyó significativamente en cuanto a producción científica ya que esta IES en el quinquenio 2003-2008 no contaba con ninguna publicación indexada a Scopus.

En la figura 5 se presentó la comparación del número de publicaciones realizadas por cada una de las diez mejores Instituciones de Educación Superior (IES) del Ecuador indexadas a Scopus durante los períodos 2003-2008 y 2013-2017. Esta comparación se la realizó con la finalidad de determinar si la reforma de educación superior de 2008 influyó significativamente en cuanto a la producción científica de cada una de las IES del Ecuador. Con base en los resultados se aprecia que esta reforma influyó positiva y significativamente en la producción científica de estas IES. Esta afirmación se la puede corroborar al observar los datos correspondientes a la Universidad de Especialidades Espíritu Santo (UEES), la Universidad de las Américas (UDLA) y la Universidad de las Fuerzas Armadas (ESPE). Estas IES en el período 2003-2008 no constaban en el SIR Iber pues tenían una producción científica igual a cero. Esta realidad cambia para el período 2013-2017, pues la Universidad de las Fuerzas Armadas termina posicionándose en primer lugar con un total de 884 publicaciones desplazando a la Universidad San Francisco de Quito. En cuanto a la UEES y la UDLA su producción científica aumentó y para este período el número de publicaciones sería de 222 y 245 respectivamente.

Licencia Creative Commons Atribución 4.0 Internacional (CC BY 4.0)

Revista Cátedra, 3(2), pp. 126-149, mayo-agosto 2020. e-ISSN: 2631-2875

https://doi.org/10.29166/10.29166/catedra.v3i2.2183 


\section{Conclusiones}

Los datos revelaron que durante el período de 2003-2008, 2006-2010 y 2009-2013 las universidades líderes en producción científica indexadas a Scopus fueron la Universidad San Francisco de Quito, la Pontificia Universidad Católica del Ecuador y la Escuela Politécnica Nacional. Por otra parte, la Universidad Espíritu Santo y la Universidad de las Américas fueron las Instituciones de Educación Superior que reflejaron una producción científica significativamente deficiente durante estos períodos. Finalmente, durante los años 2013-20017 las universidades con mayor producción científica varían en relación con los períodos anteriores. Siendo así, en primer lugar, se posiciona la Universidad de las Fuerzas Armadas, seguida la Universidad San Francisco de Quito y la Universidad Técnica Particular de Loja. Pero, la Universidad Espíritu Santo y la Universidad de las Américas se mantienen como las Instituciones de Educación Superior CON menor producción científica.

El número de publicaciones indexadas a Scopus durante los períodos 2003-2008, 2006$2010,2009-2013$ y 2013-2017 no ha permitido que las universidades ecuatorianas puedan posicionarse dentro de las 100 mejores a nivel de Iberoamérica y Latinoamericano. No obstante, se ha evidenciado el avance que han tenido dentro del ranking de universidades establecido por SIR. A pesar de que Instituciones de Educación Superior como la Universidad San Francisco de Quito y la Pontificia Universidad Católica del Ecuador no han cambiado su estatus aún con el aumento de publicaciones. Las ocho universidades restantes han subido drásticamente dentro del listado de universidades iberoamericanas. Según los últimos resultados del 2017 la universidad líder en publicaciones a nivel nacional es la Universidad de las Fuerzas Armadas destronando así a la Universidad San Francisco de Quito.

Con el nuevo modelo de Educación Superior implementado a partir de la reforma de Educación Superior de 2008 la producción científica de las Universidades a nivel nacional mejoró notablemente. Siendo así la Universidad San Francisco de Quito de un total de 200 publicaciones incrementó a 636 publicaciones registradas en Scopus. De igual manera la Pontificia Universidad Católica del Ecuador de 118 publicaciones aumentó a 571 publicaciones. La Escuela Politécnica Nacional de 113 publicaciones incrementó a 691 publicaciones indexadas a Scopus. En cuanto a las Universidades con menor número de publicaciones científicas antes de la reforma de 2008 se encuentran la Universidad Espíritu Santo y la Universidad de las Américas, sin embargo, estas mejoran notablemente a partir del año 2009 ya que para el 2017 llegan a registrar 222 y 245 publicaciones en Scopus respectivamente. Estos datos revelan mayor incremento de producción científica lo que implica que se aborden más temas y que las IES del Ecuador sean partícipes en la generación de conocimiento científico.

\section{Agradecimientos}

Agradecemos a la MSc. Verónica Simbaña- Gallardo por la guía dada durante el proceso de investigación y redacción de este artículo.

\section{Bibliografía}

Añazco, D. (2017). Apuntes sobre la producción científica en el Ecuador.Academia.edu. Recuperado el 30 de diciembre de 2019 de https://www.academia.edu/29737989/APUNTES SOBRE LA PRODUCCI\%C3\%93 N CIENT\%C3\%8DFICA EN EL ECUADOR 
Ayala, E. (2015). La investigación científica en las universidades ecuatorianas. Anales Revista de la Universidad de Cuenca. 57, pp.61-72 Recuperado el 28 de diciembre, 2019 del https://dspace.ucuenca.edu.ec/bitstreggam/123456789/22935/1/6.pdf

Bruque, S. (2013). La producción científica en Ecuador en el contexto latinoamericano. [diapositivas]. Ecuador: Prometeo. Recuperado el 30 de diciembre de 2019 de: https://www.epn.edu.ec/wp-content/uploads/2017/03/Producion-cientifica-enEcuador.pdf

Cabrera, L., Salas. N, Salas. S, y Esteves, Z., (2018). Producción Científica en la universidad ecuatoriana. Revista Científica Mundo de la Investigación y el Conocimiento, 2. QuitoEcuador. Recuperado el 28 de diciembre, 2019 de https://www.recimundo.com/index.php/es/article/view/183

CACES. (2018). Política de Evaluación Institucional de Universidades y Escuelas Politécnicas en el marco del Sistema de Aseguramiento de la Calidad de la Educación Superior. $\begin{array}{llllll}\text { Recuperado el } & 17 & \text { de } & \text { enero, } & 2020 & \end{array}$ https://www.caces.gob.ec/documents/20143/714527/1.+CACES POLITICAS EVA LUACION INSTITUCIONAL 2018+\%281\%29.pdf/94194848-e043-e8f3-9a7f$\underline{\mathrm{f} 9 \mathrm{c} 4 \mathrm{f} 9 \mathrm{~b} 5 \mathrm{e} 874}$

CACES (2019). Evaluación externa con fines de acreditación de Universidades y Escuelas politécnicas. Recuperado el 16 de enero, 2020 de https://www.caces.gob.ec/web/ceaaces/institucional

Castillo, J. A.; Powell, M. A. (2019). Análisis de la producción científica del Ecuador e impacto de la colaboración internacional en el periodo 2006-2015. Revista Española de Documentación Científica, 42 (1): e225. https://doi.org/10.3989/redc.2019.1.1567

CEAACES (2013). Evaluación de la Universidad y Escuelas Politécnicas. Quito, Ecuador.

Consejo de Educación Superior (2012). [en línea]. Ecuador. Recuperado el 15 de enero de 2020 de: http://www.ces.gob.ec/

CONEA (2003). El Sistema nacional de evaluación y acreditación de la educación superior: estructura, políticas, estrategias, procesos y proyecciones. https://unesdoc.unesco.org/ark:/48223/pf0000148843

Constitución de la República del Ecuador (2008). [en línea]. Ecuador: Publicación oficial de la Asamblea Constituyente. Recuperado el 15 de enero de 2020 de: https://www.asambleanacional.gob.ec/sites/default/files/documents/old/constit ucion de bolsillo.pdf

Constitución de la República del Ecuador. Registro Oficial N 449, Montecristi- Ecuador, 20 de octubre de 2008.

DFHC. (2015). USFQ es la Universidad con Mayor Producción Científica del Ecuador, según informe SIR Iberoamérica 2015. Recuperado el 30 de diciembre de 2019, de: https://noticias.usfq.edu.ec/2015/03/USFQMayorProduccionCientificaEcuador.html

El Comercio. (2020). [en línea].15 universidades ecuatorianas en el ranking mundial. Recuperado el 30 de diciembre de 2019, de:https://www.elcomercio.com/actualidad/universidades-ecuatorianas-rankingmundial-educacion.html 
Elsevier. (2016). Guía del usuario. Recuperado el 28 de diciembre de 2019 de: https://www.recursoscientificos.fecyt.es/sites/default/files/guia-del-usuario.pdf

https://webs.ucm.es/centros/cont/descargas/documento19197.pdf

Ley Orgánica de Educación Superior. Registro Oficial No 77 de 15 -may-2000. QuitoEcuador, 12 de oct. de 2010. Recuperado el 16 de enero, 2020 de: http://www.ces.gob.ec/index.php?option=com phocadownload\&view=category\&i $\underline{\mathrm{d}=11 \text { :ley-organica-de-educacion-superior\&Itemid=137 }}$

Ley Orgánica de Educación Superior (2010a). [en línea]. Ecuador. Recuperado el 15 de enero de 2020 de: https://www.educacionsuperior.gob.ec/wpcontent/uploads/downloads/2014/03/LEY ORGANICA DE EDUCACION SUPERIO R LOES.pdf

Ley Orgánica de Educación Superior. Registro Oficial N² 298, Quito-Ecuador, 12 de octubre de 2010. Recuperado el 16 de enero, 2020 de: http://www.ces.gob.ec/index.php?option=com phocadownload\&view=category\&i $\mathrm{d}=11$ :ley-organica-de-educacion-superior\&Itemid=137

Méndez, S. García, R. y Ortega, J. (2016). La producción científica en la universidad ecuatoriana: Una perspectiva sobre la difusión de conocimiento Nacional, regional y mundial. Revista Ciencias Pedagógicas e Innovación,4(2),54-59.Recuperado el 30 de diciembre de 2019 de: https://incyt.upse.edu.ec/pedagogia/revistas/index.php/rcpi/article/view/136/1 $\underline{28}$

PACHECO OLEA, Leonidas Augusto; PACHECO-MENDOZA. Rosa, (2015). Evolución de la educación superior en el Ecuador. La Revolución Educativa de la Universidad Ecuatoriana, Pacarina del Sur [En línea], 6(23), abril-junio, 2015. ISSN: 2007-2309. Recuperado 16 de 16 Enero de 2020 de http://www.pacarinadelsur.com/home/amautas-y-horizontes/1128-evolucionde-la-educacion-superior-en-el-ecuador-la-revolucion-educativa-de-launiversidad-ecuatoriana

Pacheco, S., Zúñiga, X., Ochoa, C. Mayorga, A., y Coka, J., (2019). Análisis de la Producción Científica de las Universidades de las Zonas 3,5 y 8 del Ecuador. Revista Espacios, 40 (12): 6. Recuperado el 28 de diciembre, 2019 de http://www.revistaespacios.com/a19v40n12/19401206.html\#iden3

Piedra, Y., y Martínez, A. (2007). Producción científica. Ciencias de la Información 38. (3),7278. Recuperado el 28 de diciembre, 2019 de: https://www.redalyc.org/articulo.oa?id=1814/181414861004

Reglamento de Régimen Académico (2017). [en línea]. Ecuador. Recuperado el 15 de enero de 2020 de: http://www.ces.gob.ec/lotaip/2018/Enero/Anexos\%20Procu/An-lita2-Reglamento\%20de\%20R\%C3\%A9gimen\%20Acad\%C3\%A9mico.pdf

Reiban-Barrera, R. y Vera-Cedeño, V. (2017). Análisis de la producción científica: Caso docentes de Nivelación de la Universidad de Guayaquil. Revista Publicando 4(10), 458-474.

https://revistapublicando.org/revista/index.php/crv/article/view/450/pdf 450

Rivera-García, C., Espinosa-Manfugás, J., y Valdés-Bencomo, Y. (2017). La investigación científica en las universidades ecuatorianas.: Prioridad del sistema educativo

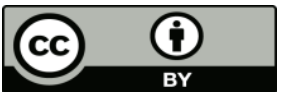

Licencia Creative Commons Atribución 4.0 Internacional (CC BY 4.0)

Revista Cátedra, 3(2), pp. 126-149, mayo-agosto 2020. e-ISSN: 2631-2875

https://doi.org/10.29166/10.29166/catedra.v3i2.2183 
vigente. Revista Cubana de Educación Superior, 36(2), 113-125. Recuperado en 17 de enero de 2020, de http://scielo.sld.cu/scielo.php?script=sci arttext\&pid=S0257$\underline{43142017000200011 \& \operatorname{lng}=\mathrm{es} \& \ln g=\mathrm{es}}$.

Rojas, Jaime E. (2011). Reforma universitaria en el Ecuador. Etapa de transición. Innovación Educativa, 11(57),59-67. Recuperado el 16 de enero de 2020 de: https://www.redalyc.org/articulo.oa?id=1794/179422350008

SENESCYT (2019). Subsecretaría de Educación, Ciencia, Tecnología e Innovación. Recuperado el 16 de enero, 2020 de https://www.educacionsuperior.gob.ec/lasecretaria/

SIR. (2010). Ranking Iberoamericano SIR 2010 3. Recuperado el 28 de diciembre, 2019 de: https://webs.ucm.es/centros/cont/descargas/documento19197.pdf

SIR. (2012). Ranking Iberoamericano SIR 2012 (3era edición).Recuperado el 28 de diciembre, 2019 de: https://www.utmachala.edu.ec/archivos/siutmach/documentos/planificacion/ra nking iberoamericano 2012.pdf

SIR. (2015). SIR Iber 2015. Recuperado el 28 de diciembre, 2019 de: https://imagenes.universia.net/gc/net/files/2015/3/9/sir-iber-2015-he.pdf

SIR. (2019). SIR Iber 2019. Recuperado el 28 de diciembre, 2019 de:http://www.elprofesionaldelainformacion.com/documentos/SIR Iber 2019.pd $\underline{\mathrm{f}}$

\section{Autores}

JORGE BALLADARES-BURGOS Es Doctor en Formación del Profesorado y TIC por la Universidad de Extremadura de España.

Actualmente es docente e investigador en la Universidad Andina Simón Bolívar -sede Ecuador.

ÁNGELA GARCÍA- NARANJO actualmente es estudiante de sexto semestre de la Carrera de Pedagogía de la Lengua y Literatura de la Universidad Central del Ecuador. Además, se encuentra cursando el penúltimo nivel de inglés para obtener la suficiencia B2 en el Centro de Educación Continua de la Escuela Politécnica Nacional.

CRISTINA GRANDA- VILLAMAR actualmente es estudiante de sexto semestre de la Carrera de Pedagogía de la Lengua y la Literatura de la Universidad Central del Ecuador. 